\title{
Editorial
}

\section{Lung Imaging in the Acute Respiratory Distress Syndrome}

The Acute Respiratory Distress Syndrome (ARDS) is a devastating and very complex disease that has been described in 1967. ARDS is under the spotlight with the recent pandemic H1N1 A influenza flu. ARDS is now a term which has been released in the media all over the world. Since its original description, an enormous amount of experimental and clinical works in all fields of research has been performed. In spite of this, no specific major advance has been made and the mortality remains high and stable. The only intervention that has been proven to be beneficial to patient outcome is the limitation of volumes and pressures applied across the lung with the mechanical ventilation.

Lung imaging is a cornerstone tool in ARDS. The definition of ARDS includes bilateral infiltrates on chest-X-ray. This not specific finding has been retained because it is sensitive to detect the risk for ARDS. Indeed, the currently approved definition of ARDS, stemming from a consensus experts conference, is an operating definition. It has been reported that, as compared with CT scan, the presence of diffuse infiltrates on a frontal chest-X-ray may correspond to diffuse, patchy or lobar loss of aeration on the CT scan. We got dramatic insights into the pathogenesis of ARDS with the CT scan thanks to the pioneering works of Prof Luciano Gattinoni in Milan, Italy. The two main drawbacks of current CT scan technology are that it is not available at the bedside and the functional informations, which are crucial to set the ventilator and are provided by the density histogram across different lung regions, are cumbersome and required time to be achieved. Furthermore, the resolution is not at the alveolar level.

In this special issue, the authors attempted to provide the readers with the last data pertaining to the use of various lung imaging modalities in ARDS. I would like to thank warmly all the contributors, which are both colleagues and friends, who made an outstanding job to clearly explain complex techniques, underline the advantages and limitations of each of them and draw the future of the research in lung imaging in this field.

\section{Claude Guérin}

(Guest Editor)

Service de Réanimation Médicale

Hôpital de la Croix-Rousse

103 Grande Rue de la Croix Rousse 69004, Lyon France

E-mail: claude.guerin@chu-lyon.fr

(C) Claude Guérin; Licensee Bentham Open.

This is an open access article licensed under the terms of the Creative Commons Attribution Non-Commercial License (http: //creativecommons.org/licenses/by-nc/ $3.0 /$ ) which permits unrestricted, non-commercial use, distribution and reproduction in any medium, provided the work is properly cited. 